\title{
AN APPROXIMATION PROPERTY RELATED TO $M$-IDEALS OF COMPACT OPERATORS
}

\author{
RAFAEL PAYÁ AND WEND WERNER
}

(Communicated by William J. Davis)

\begin{abstract}
We investigate a variant of the compact metric approximation property which, for subspaces $X$ of $c_{0}$, is known to be equivalent to $K(X)$, the space of compact operators on $X$, being an $M$-ideal in the space of bounded operators on $X, L(X)$. Among other things, it is shown that an arbitrary Banach space $X$ has this property iff $K(Y, X)$ is an $M$-ideal in $L(Y, X)$ for all Banach spaces $Y$ and, furthermore, that $X$ must contain a copy of $c_{0}$. The proof of the central theorem of this note uses a characterization of those Banach spaces $X$ for which $K(X)$ is an $M$-ideal in $L(X)$ obtained earlier by the second author, as well as some techniques from Banach algebra theory.
\end{abstract}

A closed subspace $J$ of a Banach space $X$ is called an $M$-ideal iff the dual space $X^{*}$ is the $l_{1}$-direct sum of the annihilator $J^{0}$ and some closed subspace, a notion that has been introduced by Alfsen and Effros in order to unify certain aspects of the theory of $C^{*}$-algebras and $A(K)$-spaces [1]. Though not using this terminology, Dixmier had already proved in 1950 [6] that, for any Hilbert space $H, K(H)$ is an $M$-ideal in $L(H)$, and since the early 70 s the question of which Banach spaces $X$ the space $K(X)$ is an $M$-ideal for in $L(X)$ has been of general interest for various reasons (for a brief survey on the connections to the theory of Banach spaces see, e.g., the introduction to [12] or [9], and for some applications consult the appropriate references in [13]). So it was shown, for example in [8], that $X$ with $K(X)$ being an $M$-ideal of $L(X)$ necessarily must possess the compact metric approximation property (CMAP), and in [4] it turned out that for subspaces of $l_{p}, 1<p<\infty$, the CMAP (here, one can dispose of the letter " $M$ ") already ensures that property.

As a matter of fact [13], $K(X)$ is an $M$-ideal in $L(X)$ iff $X$ satisfies the following strong version of the CMAP:

There is a net $\left(K_{\alpha}\right)$ in the unit ball $B_{K(X)}$ of $K(X)$ converging to the identity strongly and satisfying

$$
\lim \sup \left\|K_{\alpha} T_{1}+\left(\mathrm{Id}-K_{\alpha}\right) T_{2}\right\| \leq 1
$$

for all $T_{1,2} \in B_{L(X)}$.

Received by the editors July 20, 1989 and, in revised form, February 22, 1990.

1980 Mathematics Subject Classification (1985 Revision). Primary 47D15, 46H20, 46B20. 
Meanwhile, it is known [12] that for subspaces $X$ of $c_{0}$ the CMAP also entails that $K(X)$ is an $M$-ideal in $L(X)$ (here, the question whether " $M$ " will survive is still in doubt) and that, furthermore, these spaces satisfy the stronger condition

$$
\lim \sup _{\alpha} \sup _{\left\|x_{1,2}\right\| \leq 1}\left\|K_{\alpha} x_{1}+\left(\mathrm{Id}-K_{\alpha}\right) x_{2}\right\| \leq 1
$$

for a net $\left(K_{\alpha}\right)$ in $B_{K(X)}$ with the identity as a strong limit.

The starting point of the present investigation is the problem of relating condition (B) to $M$-ideals of compact operators. The central result of this note states that a Banach space $X$ satisfies condition (B) iff $K(Y, X)$ is an $M$-ideal in $L(Y, X)$ for all spaces $Y$.

In addition, we show that an infinite-dimensional space with this property necessarily contains a copy of $c_{0}$, and hence, condition (B) is strictly stronger than condition (A). After presenting a dual version of condition (B), we conclude this article by proving that no infinite-dimensional Banach space $X$ satisfies the condition

$$
K(X, Y) \text { is an } M \text {-ideal in } L(X, Y) \text { for all spaces } Y \text {. }
$$

We should remark at this point that the only examples known to the authors which actually share property (B) are those Banach spaces $X$ which are almost isometric to subspaces of $c_{0}(\Gamma)$ having the CMAP. It is an outstanding problem whether this is solely due to the lack of a good example.

We will use the notation $X_{\infty}^{2}$ or $X_{1}^{2}$ to denote the square of $X$ provided with the $l_{\infty}$ - or $l_{1}$-norm, respectively, and apply the fact that

$$
L\left(Y, X_{\infty}^{2}\right) \cong L(Y, X)_{\infty}^{2} \quad \text { and } \quad L\left(X_{1}^{2}, Y\right) \cong L(X, Y)_{\infty}^{2},
$$

as well as the analogous statement for spaces of compact operators without previous comment.

All other notation seems to be standard. We prepare for the proof of our main theorem with the following lemma, which is probably known. In the absence of an appropriate reference, we indicate a proof. (For the definition of the Arens products, the reader is referred to, e.g., [7]).

Lemma 1. Let $\mathfrak{A}$ be a Banach algebra, and suppose that the algebra $M_{n}(\mathfrak{A})$ of $n \times n$ matrices with entries in $\mathfrak{A}$ is equipped with some Banach algebra norm which generates the product topology. Then $M_{n}(\mathfrak{A})^{* *}$, endowed with any Arens product can be identified as an algebra with $M_{n}\left(\mathfrak{A}^{* *}\right)$, provided that $\mathfrak{A}^{* *}$ bears the same Arens product. Under this identification, $w^{*}$-convergence of elements in $M_{n}(\mathfrak{A})^{* *}$ is equivalent to $w^{*}$-convergence in each entry of the corresponding matrices in $M_{n}\left(\mathfrak{A}^{* *}\right)$.

In the sequel, we will provide $M_{n}\left(\mathfrak{A}^{* *}\right)$ with the Banach algebra norm given by the above identification. 
Proof. For $i, j=1,2, \ldots, n$ and $x \in M_{n}(\mathfrak{A})$, let $T_{i j}(x)$ be the entry at position $(i, j)$ in $x$. By assumption, $T_{i j}$ is a continuous linear mapping from $M_{n}(\mathfrak{A})$ onto $\mathfrak{A}$.

For $F \in M_{n}(\mathfrak{A})^{* *}$ let $\Phi(F) \in M_{n}\left(\mathfrak{A}^{* *}\right)$ be the matrix whose $(i, j)$-entry is $T_{i j}^{* *}(F)$. As a Banach space, $M_{n}(\mathfrak{A})$ is nothing but $\mathfrak{A}^{n^{2}}$, and the same holds true for $M_{n}\left(\mathfrak{A}^{* *}\right)$ and $\left(\mathfrak{A}^{* *}\right)^{n^{2}}$. This should be enough to persuade the reader that $\Phi$ is a linear bijection from $M_{n}(\mathfrak{A})^{* *}$ onto $M_{n}\left(\mathfrak{A}^{* *}\right)$ and that our claim concerning $w^{*}$-convergence is true. The fact that $\Phi$ is a homomorphism needs some care. One must check that

$$
T_{i j}^{* *}(F G)=\sum_{k=1}^{n} T_{i k}^{* *}(F) T_{k j}^{* *}(G)
$$

for $i, j=1,2, \ldots, n$ and arbitrary elements $F, G$ in $M_{n}(\mathfrak{A})^{* *}$. However, the above equation holds whenever $F, G \in M_{n}(\mathfrak{A})$, and so the proof consists in carefully passing to the bidual through standard arguments involving the wellknown continuity properties of the Arens products. Note that the same Arens product has to be used on both sides of the above relation.

Theorem 2. For a Banach space $X$, the following assertions are equivalent:

(i) For all Banach spaces $Y, K(Y, X)$ is an $M$-ideal in $L(Y, X)$.

(ii) $K\left(X_{\infty}^{2}\right)$ is an $M$-ideal in $L\left(X_{\infty}^{2}\right)$.

(iii) There is a net $\left(K_{\alpha}\right)$ in the closed unit ball of $K(X)$ converging to the identity in the strong operator topology such that

$$
\limsup \sup _{\left\|x_{1,2}\right\| \leq 1}\left\|K_{\alpha} x_{1}+\left(\operatorname{Id}-K_{\alpha}\right) x_{2}\right\| \leq 1 .
$$

Moreover, the net $\left(K_{\alpha}\right)$ in (iii) may be chosen so that $\left(K_{\alpha}^{*}\right)$ converges to the identity of $X^{*}$ in the strong operator topology of this space.

Proof. (i) $\Rightarrow$ (ii) : By assumption, $K\left(X_{\infty}^{2}, X\right)$ must be an $M$-ideal in $L\left(X_{\infty}^{2}, X\right)$ and, hence, (ii) follows from the fact that $J_{\infty}^{2}$ is an $M$-ideal in $E_{\infty}^{2}$ whenever $J$ is an $M$-ideal in the Banach space $E$.

(ii) $\Rightarrow$ (iii): By [8], $X_{\infty}^{2}$ has the compact metric approximation property, and so does $X$, being a 1 -complemented subspace. This means that there is a net $\left(S_{\alpha}\right)$ in the unit ball of $K(X)$ converging to a norm-one idempotent element $p \in K(X)^{00}$ in the $w^{*}$-topology of $L(X)^{* *}$, when the latter space is given its second Arens product ([3, Proposition 28.7]; note that "right" has to be exchanged for "left" and, consequently, the second Arens product comes into play).

Applying Lemma 1 to $\mathfrak{A}=L(X)$ and $M_{2}(\mathfrak{A})$, where the latter algebra bears the norm that appears naturally through the identification $M_{2}(\mathfrak{A})=L\left(X_{\infty}^{2}\right)$, we find that the compact operators

$$
\widehat{S}_{\alpha}:=\left(\begin{array}{cc}
S_{\alpha} & 0 \\
0 & S_{\alpha}
\end{array}\right)
$$


are converging to

$$
\hat{p}=\left(\begin{array}{ll}
p & 0 \\
0 & p
\end{array}\right) \in B_{K\left(X_{\infty}^{2}\right)^{00}}
$$

in the $w^{*}$-topology of $L\left(X_{\infty}^{2}\right)^{* *}$, and that $\hat{p}$ is idempotent with respect to the second Arens product of $L\left(X_{\infty}^{2}\right)^{* *}$. Since $M$-projections are unique among norm-one projections with a common range [8], the $M$-projection from $L\left(X_{\infty}^{2}\right)^{* *}$ onto $K\left(X_{\infty}^{2}\right)^{00}$ necessarily coincides with $L_{\hat{p}}$, the operator multiplying from the left w.r.t. second Arens product.

Let

$$
\widehat{U}_{\alpha}=\left(\begin{array}{cc}
U_{11}^{\alpha} & U_{12}^{\alpha} \\
U_{21}^{\alpha} & U_{22}^{\alpha}
\end{array}\right)
$$

be a net in $B_{K\left(X_{\infty}^{2}\right)}$, tending to $\hat{p}$ in the $w^{*}$-topology such that

$$
\underset{\alpha}{\limsup }\left\|\widehat{U}_{\alpha} \widehat{R}_{1}+\left(\widehat{\mathrm{Id}}-\widehat{U}_{\alpha}\right) \widehat{R}_{2}\right\| \leq 1 \quad \forall \widehat{R}_{1,2} \in B_{L\left(X_{\infty}^{2}\right)}
$$

(for the existence, see [13]). Using Lemma 1 once more, we conclude that the weak* and in this case also the weak limit of the nets $\left(U_{12}^{\alpha}\right)$ and $\left(U_{21}^{\alpha}\right)$ is equal to zero.

In passing to an appropriate convex combination if necessary, we therefore may suppose that both nets are converging in norm. The reader should observe that this procedure does not affect the validity of (1). Consequently, there is a net $\left(\widehat{V}_{\alpha}\right)$ in $B_{K\left(X_{\infty}^{2}\right)}$ of the form

$$
\widehat{V}_{\alpha}=\left(\begin{array}{cc}
V_{1}^{\alpha} & 0 \\
0 & V_{2}^{\alpha}
\end{array}\right)
$$

still tending to $\hat{p}$ in the $w^{*}$-topology and satisfying (1). With similar reasoning, we may pass to a net $\left(\widehat{T}_{\alpha}\right)$ in $B_{K\left(X_{\infty}^{2}\right)}$ of the form

$$
\widehat{T}_{\alpha}=\left(\begin{array}{cc}
K_{\alpha} & 0 \\
0 & K_{\alpha}
\end{array}\right),
$$

which continues to have the properties of the preceding nets.

To complete this part of the proof, put

$$
\widehat{R}_{1}=\left(\begin{array}{cc}
\operatorname{Id}_{X} & 0 \\
0 & 0
\end{array}\right) \text { and } \widehat{R}_{2}=\left(\begin{array}{cc}
0 & \operatorname{Id}_{X} \\
0 & 0
\end{array}\right),
$$

which yields

$$
\underset{\alpha}{\limsup } \sup _{\left\|x_{1,2}\right\| \leq 1}\left\|K_{\alpha} x_{1}+\left(\mathrm{Id}-K_{\alpha}\right) x_{2}\right\|=\underset{\alpha}{\lim \sup }\left\|\widehat{T}_{\alpha} \widehat{R}_{1}+\left(\widehat{\mathrm{Id}}-\widehat{T}_{\alpha}\right) \widehat{R}_{2}\right\| \leq 1,
$$

and $\left(K_{\alpha}\right)$ is what we were looking for.

(iii) $\Rightarrow$ (i): Let $Y$ be any Banach space $T \in B_{L(Y, X)}$ as well as $K_{1,2,3} \in$ $B_{K(Y, X)}$ and fix $\varepsilon>0$. For $\alpha$ sufficiently large, we have

$$
\left\|K_{\alpha} K_{i}-K_{i}\right\| \leq \frac{\varepsilon}{2} \quad i=1,2,3,
$$


as well as

$$
\sup _{\left\|x_{1,2}\right\| \leq 1}\left\|K_{\alpha} x_{1}+\left(\mathrm{Id}-K_{\alpha}\right) x_{2}\right\| \leq 1+\frac{\varepsilon}{2},
$$

and consequently, putting $K_{0}:=K_{\alpha} T$, we obtain $K_{0} \in K(Y, X)$ and

$$
\left\|K_{i}+T-K_{0}\right\| \leq\left\|K_{\alpha} K_{i}+\left(\operatorname{Id}-K_{\alpha}\right) T\right\|+\left\|K_{\alpha} K_{i}-K_{i}\right\| \leq 1+\varepsilon
$$

for $i=1,2,3$. In light of Lima's characterization of $M$-ideals [10], we are done.

Finally, let us remark that the net $\left(\widehat{U}_{\alpha}\right)$ could have been chosen right from the start so as to satisfy, additionally,

$$
\lim _{\alpha} \widehat{U}_{\alpha}^{*}=\operatorname{Id}_{X_{\infty}^{2 *}}
$$

with respect to the strong operator topology on $X_{\infty}^{2 *}$ [13], a property which remains unaltered until the very end of the proof.

By [11], the spaces characterized by Theorem 2 must be $M$-ideals in their biduals. As such, they either contain a copy of $c_{0}$ or else they are reflexive [8]. We exclude the latter possibility:

Corollary 3. An infinite-dimensional Banach space $X$ satisfying any of the conditions in the Theorem 2 contains a copy of $c_{0}$.

Proof. In view of the Bessaga-Pelcziński Theorem (see [5, Theorem V.8.] for example), we are left with constructing a sequence $\left(x_{n}\right)$ in $X$ such that

$$
\inf _{n}\left\|x_{n}\right\|>0 \text { and } \sup \left\|\sum_{i=1}^{n} \varepsilon_{i} x_{i}\right\|<\infty,
$$

where the supremum is taken over all finite sums and all choices of scalars $\varepsilon_{i}$ with $\left|\varepsilon_{i}\right|=1$.

To this end, pick a sequence $\left(\delta_{n}\right)$ of positive numbers such that

$$
\prod_{n=1}^{\infty}\left(1+2 \delta_{n}\right)<\infty \text {. }
$$

By induction, we may suppose that $x_{1}, x_{2}, \ldots, x_{n}$ have been chosen as to satisfy $\left\|x_{i}\right\|>\frac{1}{2}$ for $i=1,2, \ldots, n$ and

$$
\left\|\sum_{i=1}^{n} \varepsilon_{i} x_{i}\right\|<M_{n}
$$

for any choice of the $\varepsilon_{i}$, where $M_{n}=\prod_{i=1}^{n}\left(1+2 \delta_{i}\right)$. Let $\left(K_{\alpha}\right)$ be a net of compact operators given by assertion (iii) in the above theorem. The fact that $\left(K_{\alpha}\right)$ converges uniformly to the identity on compact subsets of $X$ and the norm inequality satisfied by $\left(K_{\alpha}\right)$ implies that we have, for sufficiently large $\alpha$ and all choices of signs,

$$
\left\|\sum_{i=1}^{n} \varepsilon_{i} x_{i}-K_{\alpha}\left(\sum_{i=1}^{n} \varepsilon_{i} x_{i}\right)\right\| \leq \delta_{n+1}
$$


and

$$
\sup _{\left\|y_{1,2}\right\| \leq 1}\left\|K_{\alpha} y_{1}+\left(\mathrm{Id}-K_{\alpha}\right) y_{2}\right\| \leq 1+\delta_{n+1} .
$$

Since the dimension of $X$ is infinite, $\left\|\mathrm{Id}-K_{\alpha}\right\| \geq 1$, and so there is $y \in X$ with $\|y\|=1$ and $\left\|y-K_{\alpha}(y)\right\|>\frac{1}{2}$. Taking $x_{n+1}=y-K_{\alpha}(y)$, we find

$$
\begin{aligned}
\left\|\sum_{i=1}^{n+1} \varepsilon_{i} x_{i}\right\| & \leq\left\|K_{\alpha}\left(\sum_{i=1}^{n} \varepsilon_{i} x_{i}\right)+\left(\mathrm{Id}-K_{\alpha}\right)\left(\varepsilon_{n+1} y\right)\right\|+\delta_{n+1} \\
& \leq M_{n}\left(1+\delta_{n+1}\right)+\delta_{n+1} \leq M_{n+1},
\end{aligned}
$$

thus completing the proof.

The class of Banach spaces treated in Theorem 2 enjoys certain stability properties, which we collect in the following theorem. Note that it is unknown whether the analogous result holds in the more general case where $K(X)$ is an $M$-ideal $L(X)$.

Theorem 4. The property characterized by Theorem 2 is preserved by passing to subspaces or quotients with the compact metric approximation property, as well as under the formation of injective tensor products and $c_{0}$-sums.

Proof. The fact that the above property passes to quotients and subspaces of $X$, which enjoy the CMAP, has already been observed in [12].

For the injective tensor product, let $X$ and $Y$ be spaces admitting nets $\left(S_{\alpha}\right) \subseteq B_{K(X)}$ and $\left(T_{\alpha}\right) \subseteq B_{K(Y)}$ satisfying the inequality of Theorem 2 (iii). Then $S_{\alpha} \widehat{\otimes}_{\varepsilon} T_{\alpha} \in K\left(X \widehat{\otimes}_{\varepsilon} Y\right)$, as well as $S_{\alpha} \widehat{\otimes}_{\varepsilon} T_{\alpha} \rightarrow \operatorname{Id}_{X \widehat{\otimes}_{\varepsilon} Y}$ strongly, and, identifying $X \widehat{\otimes}_{\varepsilon} Y$ canonically with a space of approximable operators from $X^{*}$ to $Y$, we easily find that

$$
\begin{aligned}
& \limsup \sup _{F_{1,2} \in B_{X \widehat{\otimes}_{\varepsilon} Y}}\left\|S_{\alpha} \widehat{\otimes}_{\varepsilon} T_{\alpha}\left(F_{1}\right)+\left(\mathrm{Id}-S_{\alpha} \widehat{\otimes}_{\varepsilon} T_{\alpha}\right)\left(F_{2}\right)\right\| \\
& \quad=\limsup \sup _{\alpha}\left\|T_{F_{1,2} \in B_{X \widehat{\otimes}_{\varepsilon} Y}} F_{1} S_{\alpha}^{*}+F_{2}-T_{\alpha} F_{2} S_{\alpha}^{*}\right\| \\
& \quad=\lim \sup _{\alpha} \sup _{F_{1,2} \in B_{X \widehat{\otimes}_{\varepsilon} Y}}\left\|T_{\alpha}\left(F_{1} S_{\alpha}^{*}+F_{2}-F_{2} S_{\alpha}^{*}\right)+\left(\mathrm{Id}-T_{\alpha}\right)\left(F_{2}\right)\right\| \\
& \quad \leq 1 .
\end{aligned}
$$

We still have to show that, whenever $\left(X_{i}\right)_{i \in \mathfrak{J}}$ is a family of Banach spaces satisfying our property, then $X:=c_{0}\left(X_{i}\right)_{i \in \mathfrak{J}}$ also does. To do so, fix for each $i \in \mathfrak{I}$ a net $\left(K_{\alpha, i}\right)_{\alpha \in A_{i}}$ of operators on $X_{i}$ furnished by Theorem 2. Denoting by $\mathfrak{F}$ the set of finite subsets of $\mathfrak{I}$ ordered by inclusion and defining $M_{F}$ to be the operator on $c_{0}\left(X_{i}\right)_{i \in \mathfrak{J}}$ that multiplies with the characteristic function of $F$ and, finally, using the pointwise order on $\mathscr{A}:=\mathfrak{F} \times \prod_{i \in \mathfrak{J}} A_{i}$ one easily checks that the net

$$
\left(K_{\alpha}\right):=\left(M_{F} \prod_{i \in \mathfrak{I}} K_{\alpha, i}\right)
$$


is in $B_{K(X)}$ and has $\operatorname{Id}_{X}$ as a strong limit. Now,

$$
\begin{aligned}
& \underset{\alpha \in \mathscr{A}}{\limsup } \sup _{\left\|x_{i}^{1,2}\right\| \leq 1}\left\|K_{\alpha}\left(x_{i}^{1}\right)+\left(\operatorname{Id}_{X}-K_{\alpha}\right)\left(x_{i}^{2}\right)\right\| \\
& \quad=\limsup _{\alpha=(F,(\alpha, i))} \sup _{\left\|x_{i}^{1,2}\right\| \leq 1} \max \left\{\max _{i \in F}\left\|K_{\alpha, i} x_{i}^{1}+\left(\operatorname{Id}_{X_{i}}-K_{\alpha, i}\right) x_{i}^{2}\right\|, \sup _{i \notin F}\left\|x_{i}^{2}\right\|\right\} \\
& \quad \leq \limsup _{\alpha=(F,(\alpha, i))} \sup _{\left\|x_{i}^{1,2}\right\| \leq 1} \sup _{i \in \mathfrak{I}}\left\|K_{\alpha, i} x_{i}^{1}+\left(\operatorname{Id}_{X_{i}}-K_{\alpha, i}\right) x_{i}^{2}\right\| \\
& \quad \leq 1
\end{aligned}
$$

and we are done.

Plainly, for all $Y$, the space $K_{w^{*}}\left(X^{*}, Y^{*}\right)$ of $w^{*}$-continuous compact operators forms an $M$-ideal in $L_{w^{*}}\left(X^{*}, Y^{*}\right)$ whenever $X$ satisfies one of the conditions in Theorem 2. We will show that more is true. First, we need a lemma:

Lemma 5. Let $X, Y, Z$ be Banach spaces, $T \in K(X, Y)$ and $S \in L\left(Y^{*}, Z^{*}\right)$. Then $S T^{*} \in K_{w^{*}}\left(X^{*}, Z^{*}\right)$, the $w^{*}$-continuous members of $K\left(X^{*}, Z^{*}\right)$.

Proof. $T^{*}$ is compact; hence, so is $S T^{*}$. We are left with showing that $S T^{*}$ is $w^{*}$-continuous.

The range of $T^{* *}$ must be contained in $X$, and so we may define $U \in$ $L(X, Y)$ to be the restriction to $Z$ of $T^{* *} S^{*}$. But $T^{* *} S^{*}$ and $U^{* *}$ are $w^{*}$ continuous operators agreeing on $Z$, and hence they must coincide. Therefore, $S T^{*}=U^{*}$ is $w^{*}$-continuous.

Theorem 6. The conditions in Theorem 2 are also equivalent to the following:

(i)' For all Banach spaces $Y, K_{w^{*}}\left(Y^{*}, X^{*}\right)$ is an $M$-ideal in $L\left(Y^{*}, X^{*}\right)$.

(ii) ${ }^{\prime} K_{w^{*}}\left(X_{1}^{* 2}\right)$ is an $M$-ideal in $L\left(X_{1}^{* 2}\right)$.

(iii) ${ }^{\prime}$ There is a net $\left(S_{\alpha}\right)$ in the closed unit ball of $K_{w^{*}}\left(X^{*}\right)$ converging to the identity in the strong operator topology such that

$$
\lim \sup \sup _{\left\|x^{*}\right\| \leq 1}\left\|S_{\alpha} x^{*}\right\|+\left\|\left(\operatorname{Id}-S_{\alpha}\right) x^{*}\right\| \leq 1 .
$$

Proof. Let $\left(K_{\alpha}\right)$ be a net, as in part (iii) of Theorem 2, which in addition has the property that $\left(K_{\alpha}^{*}\right)$ converges to $\mathrm{Id}_{X^{*}}$. An easy dualization argument yields that the operators

$$
S_{\alpha}=K_{\alpha}^{*}
$$

satisfy all the properties announced in (iii) ${ }^{\prime}$.

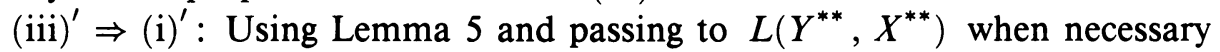
to estimate the norms of the operators involved, the proof proceeds as in the implication (iii) $\Rightarrow$ (i) of Theorem 2 .

$(\mathrm{i})^{\prime} \Rightarrow(\mathrm{ii})^{\prime}$ : Again, the proof is similar to the corresponding part in the proof of Theorem 2 . 
Let us finally show that (ii) ${ }^{\prime}$ implies (ii) of Theorem 2: Suppose that $K_{w^{*}}\left(X_{1}^{* 2}\right)$. Then $K\left(X_{\infty}^{2}\right)$ must be an $M$-ideal in $L\left(X_{\infty}^{2}\right)$, since

$$
K\left(X_{\infty}^{2}\right)=K_{w^{*}}\left(X_{1}^{* 2}\right) \subseteq L_{w^{*}}\left(X_{1}^{* 2}\right)=L\left(X_{\infty}^{2}\right) \subseteq L\left(X_{1}^{* 2}\right),
$$

and because every $M$-ideal $J \subseteq X$ remains such a thing in any intermediate space $Y$ between $X$ and $J$.

Similarly to the above, one can ascend to the higher duals of $X$ ad nauseam. We preferred to stop at this point. (For a bidual version of the above, see [12].)

We finally turn to the question of which class of Banach spaces is obtained when the order of $X$ and $Y$ in Theorem 2 is exchanged.

Proposition 7. If $K(X, Y)$ is an $M$-ideal in $L(X, Y)$ for all Banach spaces $Y$, then $X$ is finite dimensional.

Proof. Observing that $K\left(X_{1}^{2}\right)$ is an $M$-ideal in $L\left(X_{1}^{2}\right)$, we conclude that $X_{1}^{2}$ is an $M$-ideal in $X_{1}^{2 * *}$ [11]. Since $X_{1}^{2}$ contains a nontrivial $L$-summand, it follows from the $L-M$ Theorem [2, p. 24] that either $X_{1}^{2 * *}$ is two-dimensional over $\mathbb{R}$ (and we are done) or $X_{1}^{2 * *}$ contains no nontrivial $M$-ideal, that is $X_{1}^{2}$ (and hence $\left.X\right)$ must be reflexive. Consequently, $K\left(X_{1}^{2 *}\right)$ is an $M$-ideal in $L\left(X_{1}^{2 *}\right)$, and since

$$
X_{1}^{2 *}=X_{\infty}^{* 2},
$$

$X^{*}$ meets the requirements of Theorem 2. If $X^{*}$ were not finite-dimensional, Corollary 3 would produce a copy of $c_{0}$ inside $X^{*}$, contradicting the reflexivity of $X$.

Let us finally mention that the last result was found independently by $P$. Harmand and D. Werner (together with an entirely different proof).

The present work was done while the second author was visiting the University of Granada. He would like to express his gratitude toward the members of the Departamento de Análisis Matemático for their hospitality.

\section{REFERENCES}

1. E. M. Alfsen and E. G. Effros, Structure in real Banach spaces. Parts I and II. Ann of Math. 96 (1972), 98-173.

2. E. Behrends, M-structure and the Banach-Stone theorem, Lecture Notes in Math. 736. Springer, Berlin, 1979.

3. F. F. Bonsall and J. Duncan, Complete normed algebras, Springer, Berlin, 1973.

4. C.-M. Cho and W. B. Johnson, A characterization of subspaces $X$ of $l^{p}$ for which $K(X)$ is an $M$-ideal in $L(X)$. Proc. Amer. Math. Soc. 93 (1985), 466-470.

5. J. Diestel, Sequences and series in Banach spaces, Springer, Berlin, 1984.

6. J. Dixmier, Les fonctionnelles linéaires sur l'ensemble des opérateurs bornés d'un espace de Hilbert, Ann. of Math. 51 (1950), 387-408.

7. J. Duncan and S. A. R. Hosseiniun, The second dual of a Banach algebra, Proc. Royal Soc. Edinburgh 84 (1979), 309-325. 
8. P. Harmand and A. Lima, Banach spaces which are $M$-ideals in their biduals, Trans. Amer. Math. Soc. 283 (1983), 253-264.

9. D. Li, Quantitative unconditionality of Banach spaces $E$ for which $K(E)$ is an $M$-ideal in $L(E)$, Stud. Math. (to appear).

10. A. Lima, Intersection properties of balls and subspaces in Banach spaces, Trans. Amer. Math. Soc. 227 (1977), 1-62.

11. _ On M-ideals and best approximation, Indiana Univ. Math. J. 31 (1982), 27-36.

12. D. Werner, Remarks on $M$-ideals of compact operators, Quart. J. Math. Oxford (to appear).

13. W. Werner, Inner M-ideals in Banach-algebras, 1988, submitted.

Universidad de Granada, Facultad de Ciencias, Departamento de Análisis MatemáTICo, 18071 Granada, Spain

Universität-Gesamthochschule Paderborn, Fachbereich 17, Postfach 1621, 4790 Paderborn, Federal Republic of Germany 